\title{
Vibrational Anisotropy
}

\author{
Paolo Fornasini \\ University of Trento, Department of Physics, Povo (Trento), Italy \\ paolo.fornasini@unitn.it
}

\begin{abstract}
The mean square relative displacement parallel to the bond direction (parallel MSRD) is directly measured by the EXAFS Debye-Waller exponent. For crystals, the comparison of the thermal expansions measured by EXAFS and by Bragg diffraction allows one to obtain also the perpendicular MSRD. The ellipsoid of relative thermal displacements is anisotropic with respect to the bond direction for all studied systems. The quantitative study of anisotropy gives original insights on the local lattice dynamics and can be exploited to investigate the vibrational mechanism of negative thermal expansion in crystals. The basic theory is here reviewed and the results of some recent experimental studies on crystals with the diamond-zincblende structure are critically compared.
\end{abstract}

\subsection{Introduction}

The comprehension of the effects of thermal disorder on extended X-ray absorption fine structure (EXAFS) has been progressively refined since the Seventies. In the first pioneering works $[1,2]$, the Debye-Waller exponent $\sigma^{2}$ was identified as the mean square relative displacement (MSRD) parallel to the bond direction and expressed in terms of eigenfrequencies and eigenvectors of the dynamical matrix of crystals. The difference between the Debye-Waller factors of EXAFS and of diffraction due to correlation effects was stressed and the correlated Debye and Einstein models were introduced. The relevance of asymmetry in the nearest neighbour distance distribution was early thereafter recognised [3]; a parametrised model based on the cumulant expansion was introduced $[4,5]$, which proved to be particularly effective when dealing with relatively weak thermal disorder. Various theoretical treatments were developed, based on different approches [6-15].

In the last years, the increase of experimental accuracy and the progress of analysis techniques led to the possibility of detecting the difference between the local thermal expansions measured by EXAFS and by Bragg diffraction in 
crystals and to the consequent evaluation of the perpendicular MSRD [16,17]. As a result, it is now possible to reconstruct the ellipsoid of relative thermal vibrations of the absorber and backscatterer atoms, which has been found to be disc-shaped with respect to the bond direction in all systems studied up to now. The degree of anisotropy of the relative ellipsoids, which depends on the difference of correlation parallel and perpendicular to the bond, gives original insights on the local lattice dynamics and has been exploited to study the local origin of negative thermal expansion in crystals $[18,19]$.

An updated introductory treatment of thermal effects in EXAFS spectra can be found in Ref. [20]. Here, after a synthetic theoretical introduction (Sect.1.2), an account is given of recent experimental results concerning the effects of thermal vibrations in some selected semiconductors (Sect.1.3). The difference between bond-lengths measured by EXAFS and Bragg diffraction is discussed in Sect.1.4 and the applications to the study of negative thermal expansion in crystals is presented in Sect.1.5.

\subsection{Theory}

The information available from a single-scattering analysis of EXAFS spectra is intrinsically one-dimensional. For a given coordination shell, EXAFS samples an effective distribution $P(r, \lambda)=\rho(r) \exp (-2 r / \lambda) / r^{2}$, where $\rho(r)$ is the real distribution of inter-atomic distances and $\lambda(k)$ is the photo-electron mean free path (Chap. 1). For relatively weak thermal disorder, both distributions, real and effective, can be parametrised in terms of their cumulants $[4,5]$ $C_{i}$ and $C_{i}^{\prime}$, respectively. The relation between $C_{i}$ and $C_{i}^{\prime}$ can be expressed by a recursion formula [21]; in practice, the difference is generally significant only for $i=1$. The leading cumulants can be obtained by a careful analysis of EXAFS spectra, better if measured as a function of temperature [16,17]. (Note

that, due to a different convention, the cumulants of the real and effective distribution are elsewhere labelled as $C_{i}^{*}$ and $C_{i}$, respectively $\left.[16,17,21]\right)$.

We review here the connection between the cumulants $C_{i}$ of the onedimensional real distribution of distances and the structural and dynamical properties of a three-dimensional crystalline system.

Let $\boldsymbol{R}_{0}$ be the distance between absorber and backscatterer atoms ( $a$ and $b$, respectively), ideally frozen at their rest positions, and let $\boldsymbol{u}_{a}$ and $\boldsymbol{u}_{b}$ be their instantaneous vibrational displacements with respect to the rest positions, due to zero-point plus thermal energy. The instantaneous inter-atomic vector distance $\boldsymbol{r}$ can be expressed as

$$
\boldsymbol{r}=\boldsymbol{R}_{0}+\Delta \boldsymbol{u}
$$

where $\Delta \boldsymbol{u}=\boldsymbol{u}_{b}-\boldsymbol{u}_{a}$ is the relative displacement. It is convenient [22] to consider the projections of $\Delta \boldsymbol{u}$ along the bond direction $\Delta u_{\|}$and in the perpendicular plane $\Delta u_{\perp}$ (Fig. 1.1), defined by the following equations: 


$$
\Delta u_{\|}=\hat{R}_{0} \cdot \Delta \boldsymbol{u}, \quad \Delta u_{\perp}^{2}=\Delta u^{2}-\Delta u_{\|}^{2} .
$$

An EXAFS spectrum corresponds to sampling, by a large number of photoelectrons, a correspondingly large number of instantaneous configurations created by zero-point and thermal motions. The quantities measured by EXAFS are thus expressed as canonical averages.

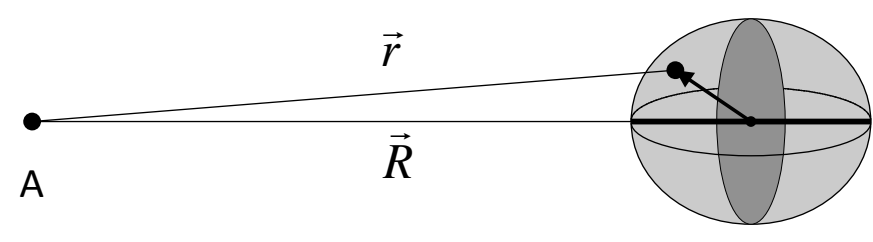

B

Fig. 1.1. Schematic representation of the relation between the equilibrium distance $\boldsymbol{R}$ and the instantaneous distance $\boldsymbol{r}=\boldsymbol{R}+\Delta \boldsymbol{u}$ between the absorber atom A and the backscatterer atom B. The ellipsoid of relative displacements is light grey. Equations (1.2) project the relative instantaneous displacement $\Delta \boldsymbol{u}$ (arrow) along the $\hat{R}$ direction (thick line) and in the perpendicular plane (dark grey).

\section{Average distance}

The average inter-atomic distance, directly measured by the first EXAFS cumulant $C_{1}$, is [17]

$$
C_{1}=\langle r\rangle \simeq R_{0}+\left\langle\Delta u_{\|}\right\rangle+\left\langle\Delta u_{\perp}^{2}\right\rangle / 2 R_{0},
$$

where $R_{0}+\left\langle\Delta u_{\|}\right\rangle=R_{c}$ is the crystallographic distance, measured by Bragg diffraction experiments. In the harmonic approximation, $\left\langle\Delta u_{\|}\right\rangle=0$ and there is no crystallographic thermal expansion. For an anharmonic crystal, the term $\left\langle\Delta u_{\|}\right\rangle$increases with temperature, and accounts for the crystallographic expansion.

In terms of the average vector positions of the absorber and backscatterer atoms, $\left\langle\boldsymbol{r}_{a}\right\rangle$ and $\left\langle\boldsymbol{r}_{b}\right\rangle$, respectively, the average distance measured by EXAFS and the crystallographic distance have different expressions:

$$
\langle r\rangle=\left\langle\left|\boldsymbol{r}_{b}-\boldsymbol{r}_{a}\right|\right\rangle \quad R_{c}=\left|\left\langle\boldsymbol{r}_{b}\right\rangle-\left\langle\boldsymbol{r}_{a}\right\rangle\right|,
$$

$R_{c}$ being the distance between average atomic positions. Eq. (1.3) shows that the average distance $\langle r\rangle$ is always larger than the crystallographic distance, owing to the presence of the perpendicular MSRD in the last term. Moreover, the difference increases with temperature, so that the thermal expansion measured by EXAFS is larger than the thermal expansion measured by Bragg 
diffraction or by dilatometric techniques. For nearest-neighbour atoms, the two distances $\langle r\rangle$ and $R_{c}$ have been recently referred to as "true" and "apparent" bond-lengths, respectively, and their temperature dependencies as true and apparent bond expansions [23]. The possibility of directly measuring the true bond expansion by EXAFS represents the solution of an old crystallographic problem, which has been for a long time faced by approximate models, such as the riding model or the TLS model [24].

\section{Parallel MSRD}

The second cumulant $C_{2}$, say the variance $\sigma^{2}$ of the distribution of distances, corresponds to a good degree of accuracy to the mean value $\left\langle\Delta u_{\|}^{2}\right\rangle$, and is now generally referred to as parallel MSRD [25]:

$$
C_{2}=\sigma^{2}=\left\langle\Delta u_{\|}^{2}\right\rangle=\left\langle\left[\hat{R} \cdot\left(\boldsymbol{u}_{b}-\boldsymbol{u}_{a}\right)\right]^{2}\right\rangle .
$$

The parallel MSRD can be expanded into the sum of three terms [1]

$$
\left\langle\Delta u_{\|}^{2}\right\rangle=\left\langle\left(\hat{R} \cdot \boldsymbol{u}_{b}\right)^{2}\right\rangle+\left\langle\left(\hat{R} \cdot \boldsymbol{u}_{a}\right)^{2}\right\rangle-2\left\langle\left(\hat{R} \cdot \boldsymbol{u}_{b}\right)\left(\hat{R} \cdot \boldsymbol{u}_{a}\right)\right\rangle .
$$

The first two terms are the independent mean square displacements (MSD) of atoms $a$ and $b$ along the bond direction, which can be calculated from the atomic displacement parameters (ADP) obtained by the refinement of Bragg diffraction patterns [26]. The third term, the displacement correlation function (DCF), depends on the correlation of atomic motions. The correlation term DCF is relatively large for the first shell and significantly smaller for the second and outer shells, reducing to zero at large distances.

In the harmonic approximation, the parallel MSRD of a crystal is connected to the eigenvalues $\omega(\boldsymbol{q}, s)$ and eigenvectors $\boldsymbol{w}(\boldsymbol{q}, s)$ of the dynamical matrix by

$$
\left\langle\Delta u_{\|}^{2}\right\rangle=\frac{1}{\mathbf{N}} \sum_{\boldsymbol{q}, s}\left\langle|Q(\boldsymbol{q}, s, t)|^{2}\right\rangle\left|\left(\frac{\boldsymbol{w}_{b}(\boldsymbol{q}, s) e^{i \boldsymbol{q} \cdot \boldsymbol{R}}}{\sqrt{m_{b}}}-\frac{\boldsymbol{w}_{a}(\boldsymbol{q}, s)}{\sqrt{m_{a}}}\right) \cdot \hat{R}\right|^{2}
$$

where $\boldsymbol{q}$ are the normal mode wavevectors, $s$ are the branch indexes, $\mathbf{N}$ is the number of primitive cells and $Q(\boldsymbol{q}, s, t)$ is the normal coordinate of mode $(\boldsymbol{q}, s)$. The parallel MSRD peculiarly depends on the phase relations between eigenvectors, on their projections on the bond direction $\hat{R}$ and on the inter-cell phase relation $e^{i \boldsymbol{q} \cdot \boldsymbol{R}}$.

Eq. (1.7) is of no practical use in EXAFS analyses. The temperature dependence of the parallel MSRD can be satisfactorily fitted to a correlated Debye Model $[1,2,27]$

$$
\sigma_{D}^{2}=\frac{3 \hbar}{\omega_{D}^{3} m} \int_{0}^{\omega_{D}} d \omega \omega \operatorname{coth} \frac{\hbar \omega}{2 k T}\left[1-\frac{\sin \left(q_{D} R\right)}{q_{D} R}\right],
$$


where $q_{D}$ is the radius of the Debye sphere and the Debye frequency $\omega_{D}$ is the only parameter of the model. The Debye frequency corresponds to a Debye temperature $\Theta_{D}=\hbar \omega_{D} / k_{B}$. For copper, as generally expected for monatomic Bravais crystals, the Debye temperatures of different coordination shells are reasonably similar [17] and in good agreement with the Debye temperatures of specific heat and of X-ray diffraction. For crystals with more than one atom per primitive cell, such as tetrahedral semiconductors, different Debye temperatures are expected for different coordination shells.

Alternatively, the temperature dependence of the parallel MSRD can be fitted to an Einstein model [2]:

$$
\left\langle\Delta u_{\|}^{2}\right\rangle=\left(\hbar / 2 \mu \omega_{\|}\right) \operatorname{coth}\left(\hbar \omega_{\|} / 2 k T\right)
$$

where $\mu$ is the reduced mass of the absorber-backscatterer atomic pair and the Einstein frequency $\omega_{\|}$is the only parameter of the model. The Einstein frequencies of different coordination shells are expected to be different also for monatomic Bravais crystals. The advantages of the Einstein model are its simplicity and the possibility of obtaining an effective force constant $k_{\|}=\mu\left(\omega_{\|}\right)^{2}$, which measures the strength of the bond between the absorber and backscatterer atoms embedded in the three-dimensional system. The force constant $k_{\|}$refers to an effective pair potential that depends on the statistically averaged influence of all the other atoms and cannot be identified with the force constant of a single-bond potential [29].

The extent of correlation of the vibrations of absorber and back-scatterer atoms can be measured by a dimensionless function of temperature [30,31]

$$
\phi_{\|}(T)=\frac{\left\langle\left(\hat{R} \cdot \boldsymbol{u}_{b}\right)^{2}\right\rangle+\left\langle\left(\hat{R} \cdot \boldsymbol{u}_{a}\right)^{2}\right\rangle-\left\langle\Delta u_{\|}^{2}\right\rangle}{2\left[\left\langle\left(\hat{R} \cdot \boldsymbol{u}_{b}\right)^{2}\right\rangle\left\langle\left(\hat{R} \cdot \boldsymbol{u}_{a}\right)^{2}\right\rangle\right]^{1 / 2}} .
$$

A value $\phi_{\|}=0$ corresponds to a completely uncorrelated motion of the two atoms. Values $\phi_{\|}=1$ and $\phi_{\|}=-1$ correspond to atomic motions perfectly in phase and in opposition of phase, respectively.

\section{Perpendicular MSRD}

The perpendicular MSRD $\left\langle\Delta u_{\perp}^{2}\right\rangle$ cannot be directly obtained from EXAFS spectra. It can however be calculated by inverting (1.3), provided $R_{c}=R_{0}+$ $\left\langle\Delta u_{\|}\right\rangle$is known from Bragg diffraction measurements.

In the harmonic approximation, the perpendicular MSRD of a crystal can be connected to the eigenvalues $\omega(\boldsymbol{q}, s)$ and eigenvectors $\boldsymbol{w}(\boldsymbol{q}, s)$ of the dynamical matrix by an expression similar to (1.7) [32]. It is worth remembering that different dynamical matrices can exist, sharing the same eigenfrequencies but with different eigenvectors [33]. The reproduction of parallel and perpendicular MSRDs, experimentally obtained from EXAFS, represents a peculiar 
test for the phase relationships between eigenvectors obtained from model calculations or ab-initio [34].

The temperature dependence of the perpendicular MSRD $\left\langle\Delta u_{\perp}^{2}\right\rangle$ is reproduced by an Einstein model [32]

$$
\left\langle\Delta u_{\perp}^{2}\right\rangle=\left(\hbar / \mu \omega_{\perp}\right) \operatorname{coth}\left(\hbar \omega_{\perp} / 2 k T\right),
$$

the only difference with respect to (1.9) being that $\mu$ is substituted for $2 \mu$ to account for the different dimensionality (projection along a line and within a plane for parallel and perpendicular MSRD, respectively). Also the perpendicular Einstein frequency can be connected to an effective force constant $k_{\perp}=\mu\left(\omega_{\perp}\right)^{2}$. Finally, a perpendicular correlation function can be defined by substituting $\left\langle\Delta u_{\perp}^{2}\right\rangle / 2$ for $\left\langle\Delta u_{\|}^{2}\right\rangle$ in the numerator of (1.10):

$$
\phi_{\perp}(T)=\frac{\left\langle\left(\hat{R} \cdot \boldsymbol{u}_{b}\right)^{2}\right\rangle+\left\langle\left(\hat{R} \cdot \boldsymbol{u}_{a}\right)^{2}\right\rangle-\left\langle\Delta u_{\perp}^{2}\right\rangle / 2}{2\left[\left\langle\left(\hat{R} \cdot \boldsymbol{u}_{b}\right)^{2}\right\rangle\left\langle\left(\hat{R} \cdot \boldsymbol{u}_{a}\right)^{2}\right\rangle\right]^{1 / 2}} .
$$

The division of $\left\langle\Delta u_{\perp}^{2}\right\rangle$ by 2 , here and in the following, projects the perpendicular MSRD along one direction.

\section{Relative vibrational anisotropy}

The knowledge of both parallel and perpendicular MSRDs allows one to reconstruct the ellipsoid of relative thermal vibrations of the pair of absorber and backscatterer atoms (assuming that the perpendicular vibrations are isotropic within the plane perpendicular to the bond). For all systems up to now investigated, the ellipsoid of relative vibrations has always been found to be anisotropic and disc-shaped, say $\left.\left\langle\Delta u_{\perp}^{2}\right\rangle / 2\right\rangle\left\langle\Delta u_{\|}^{2}\right\rangle$.

The degree of anisotropy of relative vibrations is measured by the ratio $\gamma=\left\langle\Delta u_{\perp}^{2}\right\rangle / 2\left\langle\Delta u_{\|}^{2}\right\rangle$. Since parallel and perpendicular MSRDs are characterised by different Einstein frequencies, the ratio $\gamma$ is generally temperature dependent (Sect.1.3, Fig. 1.4). A temperature-independent measure of anisotropy, corresponding to the asymptotic behaviour of $\gamma$ for $T \rightarrow \infty$, is the ratio of parallel to perpendicular effective force constants, $\xi=k_{\|} / k_{\perp}[35]$. For perfectly isotropic relative vibrations, $\xi=1$. The anisotropy of relative vibrations is a consequence of the different degree of correlation of atomic motion parallel and perpendicular to the bond.

\subsection{Experimental results on vibrational anisotropy}

The comparison of the bond thermal expansions measured by EXAFS and by Bragg diffraction has been done for a number of different systems characterized by different structures: copper [17], germanium [16] and crystals with the structures of zincblende $[19,21,28,36]$, cuprite [26], delafossite [35]. Relative 
values of both parallel and perpendicular MSRDs with respect to a reference temperature have been obtained, according to (1.3) and (1.5), as:

$$
\delta\left\langle\Delta u_{\|}^{2}\right\rangle=\delta C_{2}, \quad \delta\left\langle\Delta u_{\perp}^{2}\right\rangle=2 R_{c}\left(\delta C_{1}-\delta R_{c}\right)
$$

Absolute values of the MSRDs have been calculated by fitting Einstein correlated models (1.9) and (1.11) to the temperature dependence of the experimental data [32]. The MSRDs from EXAFS have been compared with the uncorrelated MSDs from diffraction, when available.

\subsubsection{The case of CdTe}

An example particularly suitable to illustrate the effects of thermal disorder on EXAFS of semiconductors is represented by $\mathrm{CdTe}$, for which high quality EXAFS data were measured from liquid helium to room temperature at both the $\mathrm{Cd}$ and Te K edges [19]. The availability of data measured at two different edges, at different times and different synchrotron beamlines and the comparison of results obtained by different data analysis procedures allowed a self-consistent evaluation and discussion of the uncertainty of results [37].

The coefficient of apparent thermal expansion of the $\mathrm{Cd}-\mathrm{Te}$ distance in CdTe, proportional to the lattice expansion, is negative below $T \simeq 65 \mathrm{~K}$, and above $150 \mathrm{~K}$ is nearly constant, $\alpha_{\text {app }} \simeq 4.3 \times 10^{-6} \mathrm{~K}^{-1}[38,39]$. The coefficient of true expansion, measured by EXAFS [19], is instead positive at all temperatures, $\alpha_{\text {tru }} \simeq 18 \times 10^{-6} \mathrm{~K}^{-1}$, much larger than $\alpha_{\text {app }}$ (Fig. 1.6, right).
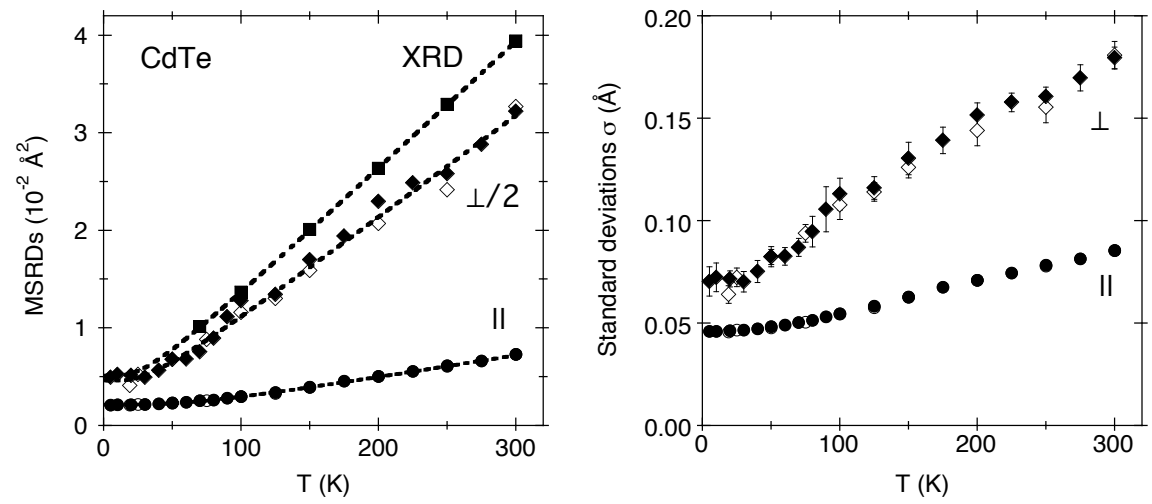

Fig. 1.2. Left panel: Parallel MSRD (circles) and halved perpendicular MSRD (diamonds) for the first shell of CdTe; solid and open symbols refer to the Te and Cd edges, respectively; the squares are the sums of the Cd and Te MSDs from [40]. The dashed lines are the best-fitting Einstein models. Right panel: Parallel and perpendicular standard deviations $\sigma_{\|}=\left[\left\langle\Delta u_{\|}^{2}\right\rangle\right]^{1 / 2}$ and $\sigma_{\perp}=\left[\left\langle\Delta u_{\perp}^{2}\right\rangle / 2\right]^{1 / 2}$. 
The first-shell parallel MSRD $\left\langle\Delta u_{\|}^{2}\right\rangle$ and the halved perpendicular MSRD $\left\langle\Delta u_{\perp}^{2}\right\rangle / 2$ of CdTe are shown in the left panel of Fig. 1.2, together with the sum of the MSDs of $\mathrm{Cd}$ and $\mathrm{Te},\left\langle\left(\hat{R} \cdot \boldsymbol{u}_{\mathrm{Cd}}\right)^{2}\right\rangle+\left\langle\left(\hat{R} \cdot \boldsymbol{u}_{\mathrm{Te}}\right)^{2}\right\rangle$, calculated from the diffraction data of [40]. The MSDs and MSRDs are well fitted by Einstein models (dashed lines in Fig. 1.2). The ratio of perpendicular to parallel MSRDs is a measure of anisotropy. By comparing the MSRDs with the sum of the MSDs, one can connect the anisotropy with the degree of correlation.

The MSRDs have the dimension of an area. They are suitable quantities for lattice dynamical interpretations, in view of their Einstein-like behaviour, which tends asymptotically to the classical linear behaviour at high temperatures. More direct physical insight is given by the standard deviations $\sigma_{\|}=\left[\left\langle\Delta u_{\|}^{2}\right\rangle\right]^{1 / 2}$ and $\sigma_{\perp}=\left[\left\langle\Delta u_{\perp}^{2}\right\rangle / 2\right]^{1 / 2}$, which have the dimension of length and directly measure the size of the relative thermal ellipsoids. The standard deviations for the first shell of CdTe are shown in the right panel of Fig. 1.2: the non negligible extent of zero-point vibrations with respect to thermal effects is evident. Standard deviations can be visually represented in real space. A pictorial comparison of the information from diffraction and from EXAFS is given in Fig. 1.3, where the absolute and relative thermal ellipsoids for the temperature of $300 \mathrm{~K}$ are shown on the same scale of the inter-atomic distance.

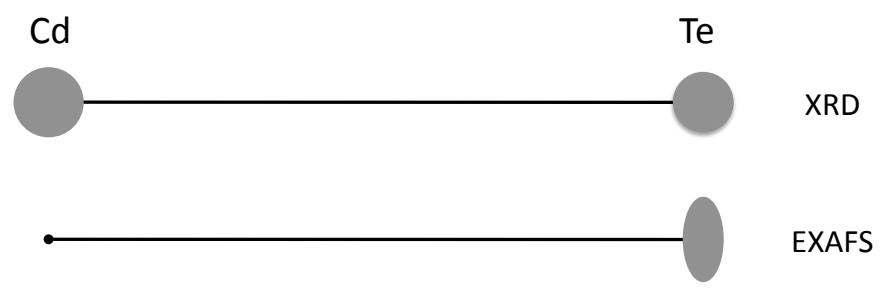

Fig. 1.3. Pictorial representation of the thermal ellipsoids for $\mathrm{CdTe}$ at $300 \mathrm{~K}$. The inter-atomic distance is $R=2.8 \AA$. The standard deviations are $\sigma_{\mathrm{Cd}}=0.15 \AA$ and $\sigma_{\mathrm{Te}}=0.13 \AA$ for the MSDs and $\sigma_{\|}=0.085 \AA$ and $\sigma_{\perp}=0.18 \AA$ for the MSRDs.

The anisotropy, measured by the ratio $\gamma=\left\langle\Delta u_{\perp}^{2}\right\rangle / 2\left\langle\Delta u_{\|}^{2}\right\rangle$, depends on temperature (Fig. 1.4, left panel). The high- $T$ asymptotic value of $\gamma$ is $\xi=$ $k_{\|} / k_{\perp}=4.6$ for CdTe. An alternative measure of anisotropy is the ratio of the standard deviations $\sigma_{\perp} / \sigma_{\|}$, which for CdTe tends asymtpotically to $\xi^{1 / 2}=2.14$ (also shown in Fig. 1.4, left panel).

The anisotropy of relative vibrations depends on the different degree of correlation along the bond direction and within the perpendicular plane, measured by the $\phi(T)$ functions of Eq. (1.10) and Eq. (1.12), respectively. Since the experimental values of MSRD and MSD had been measured at different temperatures, the correlation functions $\phi(T)$ have been evaluated using the best-fitting Einsten models; the results are shown in Fig. 1.4 (right panel). The 

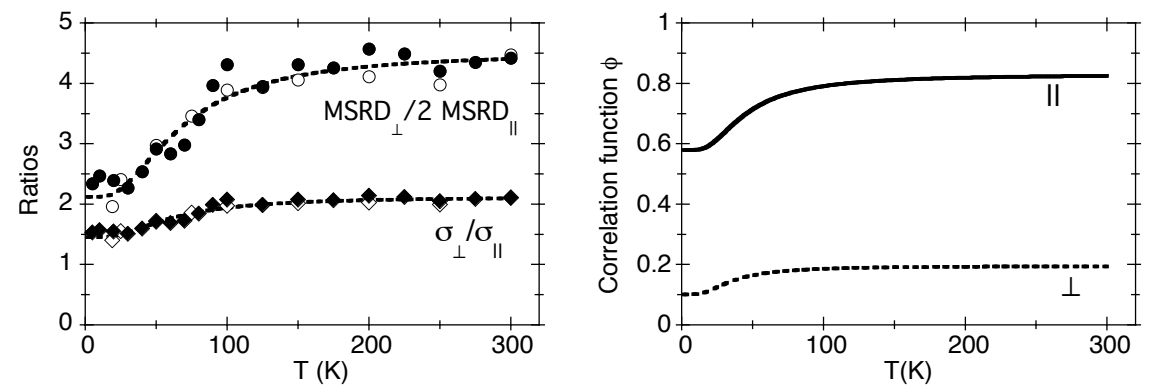

Fig. 1.4. Left panel: anisotropy of the relative vibrations in $\mathrm{CdTe}$ measured by the ratios between the MSRDs (circles) and between the standard deviations (diamonds); solid and open symbols refer to Te and $\mathrm{Cd} \mathrm{K}$ edges, respectively. Right panel: correlation functions $\phi_{\|}(T)$ (continuous line) and $\phi_{\perp}(T)$ (dashed line) calculated from the Einstein models best fitting the MSDs and MSRDs for the first shell of CdTe.

Table 1.1. Parallel and perpendicular Einstein effective force constants $k$ and anisotropy parameters $\xi=k_{\|} / k_{\perp}$ for the first shell of some selected systems, compared with ionicity and mass ratio.

\begin{tabular}{llccccc}
\hline & $\mathrm{Cu}[17]$ & $\mathrm{Ge}[16]$ & $\mathrm{GaAs}[28]$ & $\mathrm{InP}[36]$ & $\mathrm{CdTe}[19]$ & $\mathrm{CuCl}[21]$ \\
\hline Ionicity [41] & 0 & 0 & 0.31 & 0.421 & 0.675 & 0.746 \\
$M_{h} / M_{l}$ & 1 & 1 & 1.07 & 3.71 & 1.421 & 1.791 \\
\hline$k_{\|}\left(\mathrm{eV} / \AA^{2}\right)$ & 3.2 & 8.5 & 7.02 & 6.65 & 3.71 & 1.4 \\
$k_{\perp}\left(\mathrm{eV} / \AA^{2}\right)$ & 2.6 & 2.9 & 1.85 & 1.11 & 0.81 & 0.26 \\
$\xi=k_{\|} / k_{\perp}$ & 1.2 & 2.9 & 3.8 & 6.0 & 4.6 & 5.4 \\
\hline
\end{tabular}

high-temperature asymptotic value for the parallel correlation, $\phi_{\|}=0.82$, is significantly higher than the value for the perpendicular correlation, $\phi_{\perp}=0.2$.

\subsubsection{Comparison of diamond and zinblende structures}

The possibilities offered by the study of parallel and perpendicular MSRDs are highlighted by the comparison of different crystals with the diamond or zincblende structure.

\section{First coordination shell}

The nearest-neighbours effective force constants $k_{\|}$and $k_{\perp}$ and the anisotropy parameter $\xi=k_{\|} / k_{\perp}$ are listed in Table 1.1 as a function of both the ionicity [41] and the ratio of the masses of the heavier to the lighter atom, $M_{h} / M_{l}$. Copper is added in the first column for comparison. 

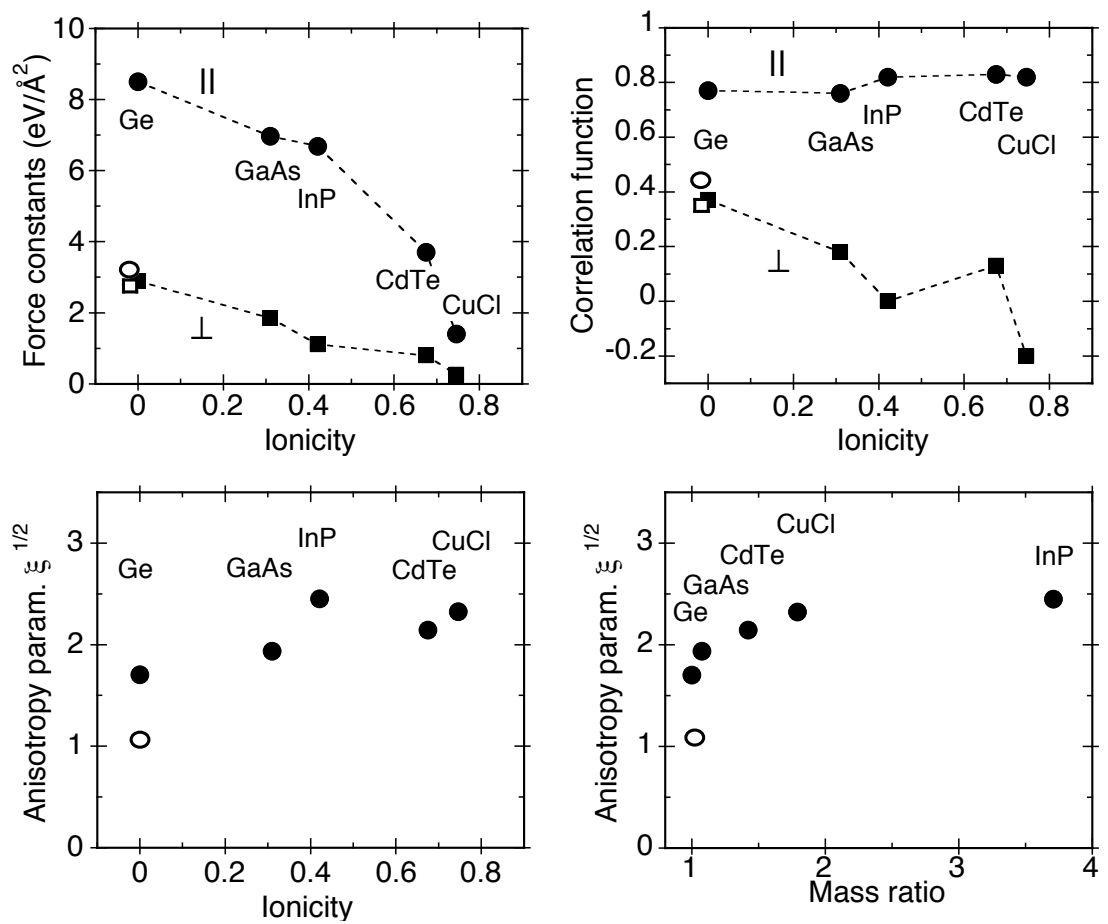

Fig. 1.5. Results for the first shell of crystals with the diamond or zincblende structure. Top panels: parallel (solid circles) and perpendicular (solid squares) force constants (left) and correlation function at $300 \mathrm{~K}$ (right), plotted as a function of ionicity; the dashed lines are a guide to eye. Bottom panels: anisotropy parameter $\xi^{1 / 2}$ as a function of both ionicity (left) and of mass ratio (right). In all panels, the open symbols are the values for $\mathrm{Cu}$.

For the diamond-zincblende crystals, a clear correlation exists between ionicity and the effective force constants: both parallel and perpendicular force constants decrease when the ionicity increases (Fig. 1.5, top left). For the tetrahedral semiconductors, the parallel force constants (solid circles) are much larger and significantly more dependent on ionicity than the perpendicular force constants (solid squares). For copper, the difference between parallel and perpendicular force constant is instead quite small (open symbols). The first-shell parallel force constant is much larger in germanium than in copper. This difference, which decreases when the ionicity increases in the other tetrahedral semiconductors, can be attributed to the difference between the totally or partially covalent bonds of tetrahedral semiconductors and the metallic bond of copper. The first-shell perpendicular force constant is instead very similar in $\mathrm{Ge}$ and in $\mathrm{Cu}$, and, for the other tetrahedral semiconductors, still decreases when ionicity increases. This behaviour can be qualitatively attributed to the open structure of semiconductors, which favours the per- 
pendicular relative vibrations of neighbouring atoms in spite of the relative bond rigidity. The smaller lateral rigidity of the metallic bond with respect to the covalent bond is compensated by the closer packing of the fcc structure.

The anisotropy parameter $\xi^{1 / 2}$ exhibits an average positive trend as a function of ioinicity (Fig. 1.5, bottom left). The scattering of data with respect to a smooth behaviour can be correlated to the different mass ratios $M_{h} / M_{l}$ of the studied compounds: the larger the mass ratio, the larger the anisotropy (Fig. 1.5, bottom right). For copper, the relative thermal vibrations are only weakly anisotropic, $\xi^{1 / 2} \simeq 1.1$ (open circles).

The parallel and perpendicular correlation functions $\phi(T)$ evaluated for $T=300 \mathrm{~K}$ are shown in Fig. 1.5 (top right). The parallel correlation (solid circles) is stronger than the perpendicular correlation (solid squares), and quite independent of ionicity, while the perpendicular correlation exhibits an average decreasing trend. For copper, the parallel correlation (open circle) is only slightly larger than the perpendicular correlation (open square).

The anisotropic behaviour of relative atomic vibrations for some systems has been recently reproduced by the ab initio calculations performed by Vila et al. [34]. The calculated values of the asymptotic ratios

$$
\lim _{T \rightarrow \infty}\left\langle\Delta u_{\perp}^{2}\right\rangle /\left\langle\Delta u_{\|}^{2}\right\rangle=2 \xi
$$

are 2.36 for $\mathrm{Cu}$ at $500 \mathrm{~K}$ and 7.2 for diamond lattices at $600 \mathrm{~K}$, in agreement with the experimental values: 2.2 for $\mathrm{Cu}$, from 5.8 and 12 for tetrahedral semiconductors (Table 1.1).

\section{Outer coordination shells}

Further information can be obtained from the analysis of the parallel MSRD of the outer coordination shells.

The parallel effective force constants $k_{\|}$and the Debye temperatures $\Theta_{D}$ for the different pairs of absorber-backscatterer atoms are listed in Table 1.2 and Table 1.3, respectively. In the Debye model for the tetrahedral semiconductors, an extended first Brillouin zone has been considered.

For copper, the Debye temperatures of the different shells are very similar, while for the tetrahedral semiconductors the Debye temperatures are strongly dependent on the shell, the most significant difference being between the first and the second shell (Table 1.3). The differences of force constants between the different shells are still stronger for tetrahedral semiconductors, and not negligible even for copper (Table 1.2).

The values of the parallel and perpendicular correlation functions for the first shell and of the parallel correlation function for the outer shells, evaluated at $T=300 \mathrm{~K}$, are listed in Table 1.4. For $\mathrm{Cu}$ and $\mathrm{CdTe}$, good quality experimental MSD data are available in the literature [40,42,43]. For GaAs and Ge we refer to theoretical calculations $[44,45]$. For InP, the perpendicular MSRD values are quite scattered, and the comparison is made with the 
Table 1.2. Parallel effective force constants $k_{\|}$(in $\mathrm{eV} / \AA^{2}$ ) for the first and outer shells of different crystals. For the 2nd shells of binary compounds, the first and second lines refer to the lightest pair $(\mathrm{Ga}-\mathrm{Ga}, \mathrm{Cd}-\mathrm{Cd})$ and to the heaviest pair (AsAs, Te-Te, In-In), respectively.

\begin{tabular}{cccccc} 
Shell & $\mathrm{Cu}[17]$ & $\mathrm{Ge}[16]$ & $\mathrm{GaAs}[28]$ & $\mathrm{InP}[36]$ & $\mathrm{CdTe}[19]$ \\
\hline 1 & 3.20 & 8.15 & 7.02 & 6.65 & 3.71 \\
2 & 1.97 & 2.48 & 2.30 & & 0.91 \\
& & & 2.68 & 2.09 & 1.29 \\
3 & 2.40 & 2.18 & 1.75 & 1.74 & 0.85 \\
4 & 2.33 & & & & \\
\hline
\end{tabular}

Table 1.3. EXAFS Debye temperatures (in $K$ ) for the first and outer shells of different crystals. For the 2nd shells of binary compounds, the first and second lines refer to the lightest pair (Ga-Ga, Cd-Cd) and to the heaviest pair (As-As, Te-Te, In-In), respectively.

\begin{tabular}{cccccc} 
Shell & $\mathrm{Cu}[17]$ & $\mathrm{Ge}[16]$ & $\mathrm{GaAs}[28]$ & $\mathrm{InP}[36]$ & $\mathrm{CdTe}[19]$ \\
\hline 1 & 328 & 460 & 402 & 416 & 228 \\
2 & 283 & 299 & 230 & & 114 \\
& & & 240 & 171 & 126 \\
3 & 322 & 290 & 195 & 203 & 105 \\
4 & 321 & & & & \\
\hline
\end{tabular}

Table 1.4. Correlation functions $\phi_{\|}$and $\phi_{\perp}$ at $T=300 \mathrm{~K}$ for different crystals. For the 2nd shells of binary compounds, the first and second lines refer to the lightest pair (Ga-Ga, Cd-Cd) and to the heaviest pair (As-As, Te-Te, In-In), respectively.

\begin{tabular}{cccccccc} 
& Shell & $\mathrm{Cu}[17]$ & $\mathrm{Ge}[16]$ & GaAs $[28]$ & $\mathrm{InP}[36]$ & $\mathrm{CdTe}[19]$ & $\mathrm{CuCl}[21]$ \\
\hline$\phi_{\|}$ & 1 & 0.46 & 0.77 & 0.76 & 0.82 & 0.83 & 0.82 \\
& 2 & 0.14 & 0.18 & 0.32 & & 0.33 & \\
& & & & 0.44 & 0.42 & 0.41 & \\
& 3 & 0.24 & 0 & 0.14 & 0.37 & 0.27 & \\
\hline$\phi_{\perp}$ & 1 & 0.36 & 0.37 & 0.18 & 0 & 0.13 & -0.2 \\
\hline
\end{tabular}

experimental MSD data of [46]. For $\mathrm{CuCl}$, the data are limited to the first shell, perpendicular MSRD values at $300 \mathrm{~K}$ are extrapolated from the values below $100 \mathrm{~K}$ and the comparison is made with an average of available MSD data [21]. In spite of the difficulty in assessing the accuracy of experimental and theoretical data, some qualitative properties are evident in Table 1.4. The first-shell parallel correlation is much stronger in tetrahedral semiconductors than in copper; the correlation is instead comparable for the outer shells. 


\subsection{True and apparent bond expansion}

Once the absolute values of the perpendicular MSRD of the first shell have been evaluated, one can attempt to gain a better quantitative evaluation of the difference between the true and apparent bond distances and thermal expansions, as per (1.3). In Fig. 1.6, the true and apparent bond expansions of GaAs and CdTe, evaluated with respect to the low-temperature crystallographic distance, are compared.

The values of the difference between true and apparent distances of some systems at low and room temperature are listed in Table 1.5. The differences depend on the extent of the perpendicular MSRD, and increase with the decreasing of the perpendicular force constant $k_{\perp}$ as well as with the increase of temperature. The discrepancy between true and apparent distances should be taken into account when calibrating EXAFS simulations against model systems of known crystallographic structure. When accuracies of the order of some $0.001 \AA$ are sought, a calibration can be misleading if the effect of perpendicular vibrations is neglected.

Table 1.5. Difference $C_{1}-R_{c}\left(10^{-3} \AA\right)$ between the nearest-neighbours bond distance measured by EXAFS and by Bragg diffraction at low and room temperature. (The value for Ge at $300 \mathrm{~K}$ is erroneously quoted as $8 \times 10^{-3} \AA$ in Table 3 of Ref. [37])

\begin{tabular}{rccccc}
$\mathrm{T}(\mathrm{K})$ & $\mathrm{Cu}[17]$ & $\mathrm{Ge}[16]$ & $\mathrm{GaAs}[28]$ & $\mathrm{InP}[36]$ & $\mathrm{CdTe}[19]$ \\
\hline 10 & 1.3 & 1.5 & 1.6 & 1.2 & 1.8 \\
300 & 3.8 & 3.9 & 5.8 & 9.5 & 11.5 \\
\hline
\end{tabular}
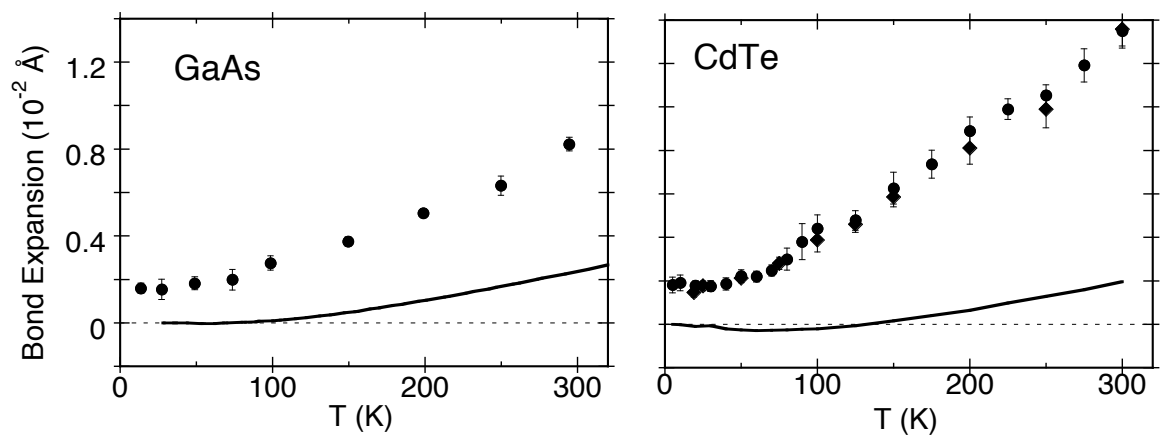

Fig. 1.6. First-shell true (symbols) and apparent (continuous line) bond expansions for GaAs (left) and CdTe (right). 


\subsection{Negative thermal expansion crystals}

The sensitivity of EXAFS to local dynamics, in particular to vibrational anisotropy, can be exploited to study the local mechanism at the origin of negative thermal expansion (NTE) in some crystals. A number of tetrahedral semiconductors (such as $\mathrm{Si}, \mathrm{Ge}, \mathrm{GaAs}, \mathrm{CdTe}, \mathrm{InP}, \mathrm{CuCl}$ [47]) exhibit lattice NTE within a restricted low-temperature interval, and some more complex framework structures are characterised by lattice NTE within extended temperature intervals [48-50].

According to the phenomenological approach of Barron [23], the net lattice expansion is the result of a competition between two contributions: a) a positive bond-stretching contribution due to the anharmonicity of the effective pair potential and $b$ ) a negative contribution due to tension effects connected to atomic movements perpendicular to the bond. When the tension effects prevail over bond stretching, the crystal exhibits NTE.

Conventional techniques, such as dilatometry and Bragg diffraction, are sensitive only to the lattice thermal expansion, and cannot distinguish the bond stretching from the tension effects. These two effects can be distinguished by complementary EXAFS measurements: the temperature dependence of the average distance $\langle r\rangle$ directly measures the positive bond expansion due to the stretching effect, while the perpendicular MSRD is connected to the tension effect.

Several crystals with different structures and characterized by different NTE strengths have been recently investigated by EXAFS: diamondzincblende structures [16,19,21,28], cuprite structures [26], delafossite structures [35]. The lattice thermal expansion is isotropic with respect to the crystallographic axes in the cubic structures (diamond-zincblende and cuprite) and anisotropic in the delafossite structure, where it is negative along the $c$ axis and positive in the perpendicular plane.

The EXAFS results confirm the possibility of distinguishing bond stretching from tension effects. For all considered systems, the bond thermal expansion, measured by the first EXAFS cumulant, is always positive; moreover, the stronger is the lattice NTE, the larger is the positive bond expansion. Within each family of isostructural compounds (diamond/zincblende, cuprite, delafossite) a stronger NTE corresponds to a smaller value of both parallel and perpendicular effective force constants as well as to a larger value of the relative vibrational anisotropy $\xi$.

According to EXAFS results, the bond stretching and tension effects can be separately quantified but are nevertheless in some way entangled. When the ionicity increases, the directional rigidity of the bond is reduced, the perpendicular effective force constant $k_{\perp}$ decreases and the perpendicular MSRD becomes larger. In a relatively open structure such as zincblende, larger vibrations perpendicular to the bond make possible also larger parallel vibrations (smaller parallel effective force constant $k_{\|}$) and a larger bond expansion. 
It is of interest to compare the directional properties of the absolute atomic vibrations, measured by Bragg diffraction, with those of the relative vibrations, measured by EXAFS. The relative thermal ellipsoids are always anisotropic with respect to the bond direction, even for cubic systems where the atomic ellipsoids are spherical for symmetry reasons. In the layered delafossite structures, $\mathrm{Cu}$ atoms belonging to a layer are linearly coordinated to two $\mathrm{O}$ atoms along the $c$ axis; the NTE along the $c$ axis is connected to the anisotropy of the thermal ellipsoids of $\mathrm{Cu}$ atoms, whose vibrations are stronger within the plane normal to the $c$ axis than along the $c$ axis. Correspondingly, also the relative vibrations of the $\mathrm{Cu}-\mathrm{O}$ atomic pair monitored by EXAFS are anisotropic, the ellipsoid being disc-shaped perpendicular to the $c$ axis [35]. The relative anisotropy measured by EXAFS for the $\mathrm{Cu}-\mathrm{O}$ pair is however larger than the anisotropy of atomic $\mathrm{Cu}$ vibrations. It seems thus that the relative vibrational anisotropy is a more general property to be connected to NTE than the absolute atomic anisotropy.

\section{References}

1. Beni, G. and Platzman, P. M. (1976) Phys. Rev. B 14, 1514-1518.

2. Sevillano, E., Meuth, H., and Rehr, J. J. (1979) Phys. Rev. B 20, 4908-4911.

3. Eisenberger, P. and Brown, G. S. (1979) Solid St. Commun. 29, 481-484.

4. Bunker, G. (1983) Nucl. Instrum. Methods Phys. Res. 207, 437-444.

5. Crozier, E. D., Rehr, J. J., and Ingalls, R. (1988) Amorphous and liquid systems. In D. C. Koningsberger and R. Prins, (ed.), X-ray Absorption, chapter 9, pp. 373-442 J. Wyley \& Sons New York.

6. Tranquada, J. M. and Ingalls, R. (1983) Phys. Rev. B 28, 3520-3528.

7. Benfatto, M., Natoli, C. R., and Filipponi, A. (1989) Phys. Rev. B 40, 96269635.

8. Frenkel, A. I. and Rehr, J. J. (1993) Phys. Rev. B 48, 585-588.

9. Fujikawa, T. and Miyanaga, T. (1993) J. Phys. Soc. Jpn. 62, 4108-4122.

10. Miyanaga, T. and Fujikawa, T. (1994) J. Phys. Soc. Jpn. 63, 1036-1052.

11. Van Hung, N. and Rehr, J. J. (1997) Phys. Rev. B 56, 43-46.

12. Yokoyama, T. (1998) Phys. Rev. B 57, 3423-3432.

13. Poiarkova, A. V. and Rehr, J. J. (1999) Phys. Rev. B 59, 948-957.

14. Katsumata, H., Miyanaga, T., Yokoyama, T., Fujikawa, T., and Ohta, T. (2001) J. Synchrotron Rad. 8, 226-228.

15. aBeccara, S., Dalba, G., Fornasini, P., Grisenti, R., Pederiva, F., Sanson, A., Diop, D., and Rocca, F. (2003) Phys. Rev. B 68, 140301.

16. Dalba, G., Fornasini, P., Grisenti, R., and Purans, J. (1999) Phys. Rev. Lett. 82, 4240-4243.

17. Fornasini, P., aBeccara, S., Dalba, G., Grisenti, R., Sanson, A., Vaccari, M., and Rocca, F. (2004) Phys. Rev. B 70, 174301.

18. aBeccara, S., Dalba, G., Fornasini, P., Grisenti, R., Rocca, F., and Sanson, A. (2003) Nucl. Intstrum. Methods Phys. Res. B 200, 237-241.

19. Abd el All, N., Dalba, G., Diop, D., Fornasini, P., Grisenti, R., Mathon, O., Rocca, F., Thiodjio Sendja, B., and Vaccari, M. (2012) J. Phys.: Condens. Matter 24, 115403. 
20. Fornasini, P. (2012) e-J. Surf. Sci. Nanotech. 10, 480-485.

21. Vaccari, M., Grisenti, R., Fornasini, P., Rocca, F., and Sanson, A. (2007) Phys. Rev. B 75, 184307.

22. Fornasini, P. (2001) J. Phys.: Condens. Matter 13, 7859-7872.

23. Barrera, G. D., Bruno, J. A., Barron, T. H. K., and Allan, N. L. (2005) J. Phys.: Condens. Matter 17, R217-R252.

24. Willis, B. T. M. and Pryor, A. W. (1975) Thermal Vibrations in Crystallography, Cambridge University Press, .

25. Fornasini, P., Monti, F., and Sanson, A. (2001) J. Synchrotron Radiat. 8, 12141220.

26. Sanson, A., Rocca, F., Dalba, G., Fornasini, P., Grisenti, R., Dapiaggi, M., and Artioli, G. (2006) Phys. Rev. B 73, 214305.

27. Böhmer, W. and Rabe, P. (1979) J. Phys. C: Solid State Phys. 12, 2465-2474.

28. Ahmed, S. I., Aquilanti, G., Novello, N., Olivi, L., Grisenti, R., and Fornasini, P. (2013) J. Chem. Phys. 139, 164512.

29. Van Hung, N. and Fornasini, P. (2007) J. Phys. Soc. Jpn 76, 084601.

30. Booth, C. H., Bridges, F., Bauer, E. D., Li, G. G., Boyce, J. B., Claeson, T., Chu, C. W., and Xiong, Q. (1995) Phys. Rev. B 52, R15745-R15748.

31. Jeong, I. K., Heffner, R. H., Graf, M. J., and Billinge, S. J. L. (2003) Phys. Rev. $B$ 67, 104301.

32. Vaccari, M. and Fornasini, P. (2006) J. Synchrotron Rad. 13, 321-325.

33. Cochran, W. (1971) Acta Cryst. A 27, 556-559.

34. Vila, F. D., Rehr, J. J., Rossner, H. H., and Krappe, H. J. (2007) Phys. Rev. B 76, 014301.

35. Ahmed, S. I., Dalba, G., Fornasini, P., Vaccari, M., Rocca, F., Sanson, A., Li, J., and Sleight, A. W. (2009) Phys. Rev B 79, 104302.

36. Schnohr, C. S., Kluth, P., Araujo, L. L., Sprouster, D. J., Byrne, A. P., Foran, G. J., and Ridgway, M. C. (2009) Phys. Rev. B 79, 195203.

37. Abd el All, N., Thiodjio Sendja, B., Grisenti, R., Rocca, F., Diop, D., Mathon, O., Pascarelli, S., and Fornasini, P. (2013) J. Synchrotron Rad. 20, 603-613.

38. Smith, T. F. and White, G. K. (1975) J. Phys. C: Solid State Phys. 8, 20312042.

39. Novikova, S. I. (1961) Soviet Phys. - Solid State 2, 2087-2089.

40. Horning, R. D. and Staudenmann, J. L. (1986) Phys. Rev. B 34, 3970-3979.

41. Martin, R. M. (1971) Phys. Rev. B 1, 4005-4011.

42. Martin, C. J. and O'Connor, D. A. (1977) J. Phys. C: Solid State Phys. 10, 3521-3526.

43. Day, J. T., Mullen, J. G., and Shukla, R. (1995) Phys. Rev. B 52, 168-176.

44. Reid, J. S. (1983) Acta Cryst. A 39, 1-13.

45. Soma, T. and Matsuo, H. (1982) Phys. Stat. Sol. (b) 111, K93-K97.

46. Saravanan, R., Mohanlal, S. K., and Chandrasekaran, K. S. (1992) Z. Kristallogr. 200, 7.

47. White, G. K. (1993) Contemp. Phys. 34, 193-204.

48. Mary, T. A., Evans, J. S. O., Vogt, T., and Sleight, A. W. (1996) Science 272, 90-92.

49. Tiano, W., Dapiaggi, M., and Artioli, G. (2003) J. Appl. Cryst. 36, 1461-1463.

50. Chapman, K. W., Chupas, P. J., and Kepert, C. J. (2005) J. Am. Chem. Soc. 127, 15630-15636. 


\section{Index}

Anisotropy, 2, 6, 8, 14

Anisotropy parameters, 9

Asymmetry, 1

Atomic displacement parameters, 4

Bond stretching, 14

Bond-lengths

apparent, 4, 13

true, 4,13

Cadmium telluride, 7, 9, 12, 13

CdTe, 7, 9, 12, 13

Copper, 9, 12

Copper chloride, 9, 12

Correlation, 4-6, 8

Correlation functions, 9, 11, 12

$\mathrm{CuCl}, 9,12$

Cumulants, 1, 2

Cuprite, 14

Debye model, 1, 4

Debye temperature, 11, 12

Debye-Waller factor, 1

Delafossites, 14

Disorder

thermal, 1

Distance

average, 3

crystallographic, 3

instantaneous, 2

Distribution

effective, 2

one-dimensional, 2

real, 2
Dynamical matrix, 1, 5

Effective distribution, 2

Effective force constants, 9, 14

Einstein model, 1, 5, 6

GaAs, 9, 12, 13

Gallium arsenide, 9, 12, 13

Germanium, 9, 12

Harmonic approximation, 3-5

Indium phosphide, 9, 12

InP, 9, 12

Ionicity, 9, 14

Mean square displacement, 4

Mean square relative displacement, 1 parallel, 4, 8

perpendicular, 5, 8

Mean-free path, 2

MSD, 4

MSRD

parallel, 1, 8

perpendicular, 2,8

Negative thermal expansion, 2, 14

Outer coordination shells, 11

Real distribution, 2

Tension effects, 14

Thermal disorder, 1

Thermal ellipsoids, 8, 15

Thermal expansion, 1, 7

apparent, 4, 13 
negative, 2,14

true, 4,13
Uncertainty evaluation, 7

Zincblende structures, 9 
